\title{
VBLAST Detection Algorithms Utilizing Soft Symbol Estimate and Noncircular CAI
}

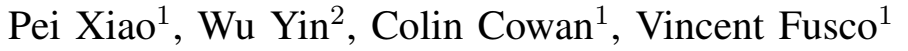 \\ ${ }^{1}$ The Institute of Electronics, Communications and Information Technology \\ Queen's University Belfast, BT3 9DT, United Kingdom \\ E-mail: \{pei.xiao, c.cowan, v.fusco\}@qub.ac.uk \\ ${ }^{2}$ Advanced Wireless Technology R \& D Center \\ ZTE Corporation, Shenzhen, China \\ E-mail: yin.wudzte.com.cn
}

\begin{abstract}
In this correspondence, we address the problem of error propagation inherent in the VBLAST detection process. To this end, two improved VBLAST schemes are proposed. The first one replaces hard decision with soft decision; whereas the other also utilizes soft symbol estimate, but in the meantime exploits the noncircular nature of the residual co-antenna interference $(\mathrm{CAI})$ and noise, it involves refining the error criterion and nulling filter. Simulation results show that both schemes outperform their conventional counterpart, and utilization of noncircular CAI significantly alleviates the error propagation problem and improves the performance of the VBLAST detection.
\end{abstract}

\section{INTRODUCTION}

The next generation wireless communication systems call for advanced signal processing techniques to support ever-increasing data rates. Multiple-input-multiple-output (MIMO) systems provide an effective means of achieving high data rate transmission without increasing the total transmission power or bandwidth for wireless systems [1]. Spatial division multiplexing (SDM) systems achieve high data rate transmission by transmitting multiple substreams simultaneously from multiple transmit antennas. The receiver also has a multiple-antenna architecture to detect spatially multiplexed substreams. For such SDM systems, maximum likelihood (ML) detection has a prohibitive complexity which grows exponentially with the number of antennas and the signal constellation size. The V-BLAST detection technique [2]-[4] offers a good tradeoff between performance and complexity. It uses a combination of linear and nonlinear detection techniques: first nulling out the interference from undetected signals, then canceling out the interference using already detected signals. However, the V-BLAST scheme suffers from the error propagation inherent in the decision feedback process. To tackle this problem, we can replicate the co-antenna intereference (CAI) components using the log-likelihood ratio (LLR) of the interfering signals and 
subtract the soft replica from the received composite-signal vector [5]. Some efficient and fast implementations of the V-BLAST algorithm have been introduced in [6], [7]. In order to approach the near-optimal performance, this successive interference cancellation (SIC) based detection scheme is complemented by maximum likelihood detection in [8] as well as lattice and list reduction scheme in [9]. An improved Turbo-MIMO detection scheme using soft-input, soft-output, and soft-feedback is presented in [10], where the authors propose to make the symbol decision by minimizing the power of the interference plus noise, given a priori probabilities of undetected layer symbols and a posteriori probabilities of past detected layer symbols.

In this correspondence, we show that the performance of the VBLAST detection can be optimized by modifying the error function after subtracting the CAI using their soft symbol estimates, a refined nulling filter is then applied to remove the residual interference. The noncircular property of the residual CAI and noise is exploited in order to improved performance of VBLAST detection. The remainder of this paper is organized as follows. The system model is described and the conventional VBLAST detection scheme is briefly reviewed in Section II. Two variants of improved VBLAST schemes are proposed in Section III and Section IV, respectivey. Numerical results are presented in Section V to compare the performance of different techniques. Finally, conclusions are drawn in Section VI.

The following notations are used: $(\cdot)^{\mathcal{T}}$ denotes matrix transpose, $(\cdot)^{\mathcal{H}}$ matrix conjugate transpose, $(\cdot)^{*}$ matrix conjugate, E[·] expectation, and $\mathbf{I}_{N}$ an $N \times N$ identity matrix.

\section{SySTEM MODEL}

We consider a MIMO system with $N$ transmit antennas and $M$ receive antennas. First, a binary input bit sequence is mapped to a complex valued symbol sequence $\mathbf{d}=\left[d_{1}, d_{2}, \ldots, d_{N}\right]^{\mathcal{T}}$, where each element is selected from a finite set or constellation alphabet and transmitted by different antenna. The received signal can be expressed as

$$
\mathbf{r}=\mathbf{H d}+\mathbf{u}=\sum_{n=1}^{N}(\mathbf{H})_{n} d_{n}+\mathbf{u} \in \mathbb{C}^{M \times 1}
$$

where $\mathbf{r}=\left[\begin{array}{llll}r_{1} & r_{2} & \ldots & r_{M}\end{array}\right]^{\mathcal{T}}$ is the received signal vector; $\mathbf{u}=\left[\begin{array}{llll}u_{1} & u_{2} & \ldots & u_{M}\end{array}\right]^{\mathcal{T}}$ denotes the complex additive white Gaussian noise vector with zero mean and covariance matrix $\sigma_{u}^{2} \mathbf{I}_{M}$. The channel matrix $\mathbf{H} \in \mathbb{C}^{M \times N}$ contains the complex channel gains, and can be formed as $\mathbf{H}=\left[\begin{array}{cccc}H_{11} & H_{12} & \ldots & H_{1 N} \\ H_{21} & H_{22} & \ldots & H_{2 N} \\ \vdots & \vdots & \ddots & \vdots \\ H_{M 1} & H_{M 2} & \ldots & H_{M N}\end{array}\right]$, where $H_{p q}$ is the complex channel gain between the $p$ th receive antenna and the $q$ th transmit antenna. The data symbols are assumed to be uncorrelated and have zero mean and unit energy, i.e., $\mathrm{E}\left[\mathbf{d d}^{\mathcal{H}}\right]=\mathbf{I}_{N}$. The vector $(\mathbf{H})_{n}$ is the $n$th column of $\mathbf{H}$. 
Let the ordered set $S \equiv\left\{k_{1}, k_{2}, \ldots, k_{N}\right\}$ be a permutation of the integer $1,2, \ldots, N$ specifying the order in which components of the transmitted symbol vector $\mathbf{d}$ are detected. The conventional VBLAST detection algorithm can be summarized as follows

$$
\begin{aligned}
k_{i} & =\arg \min _{j \notin\left\{k_{1}, \ldots, k_{i-1}\right\}}\left\|\left(\mathbf{G}_{i}\right)_{j}\right\|^{2} \\
\mathbf{r}_{i} & =\mathbf{r}_{i-1}-\hat{d}_{k_{i-1}}(\mathbf{H})_{k_{i-1}} \\
\mathbf{G}_{i} & =\left(\mathbf{H}_{\overline{k_{i-1}}}^{\mathcal{H}} \mathbf{H}_{\overline{k_{i-1}}}+\sigma_{u}^{2} \mathbf{I}\right)^{-1} \mathbf{H}_{\overline{k_{i-1}}}^{\mathcal{H}} \\
\mathbf{w}_{k_{i}} & =\left[\mathbf{G}_{i}\right]_{k_{i}} \\
y_{k_{i}} & =\mathbf{w}_{k_{i}}^{\mathcal{H}} \mathbf{r}_{i} \\
\hat{d}_{k_{i}} & =Q\left(y_{k_{i}}\right) \\
i & =i+1
\end{aligned}
$$

where $Q(\cdot)$ denotes the slicing operation appropriate for the constellation in use; $\left[\mathbf{G}_{i}\right]_{j}$ is the $j$ th row of $\mathbf{G}_{i}$; $\mathbf{H}_{\overline{k_{i-1}}}$ denotes the matrix obtained by zeroing columns $k_{1}, k_{2}, \ldots, k_{i-1}$ of $\mathbf{H}$. The above recursive procedure is initialized with $\mathbf{r}_{1}=\mathbf{r}$ and $\mathbf{G}_{1}=\left(\mathbf{H}^{\mathcal{H}} \mathbf{H}+\sigma_{u}^{2} \mathbf{I}\right)^{-1} \mathbf{H}^{\mathcal{H}}$. Note that the algorithm expressed by (3) is the minimum mean square error (MMSE) variant of the original VBLAST algorithm proposed in [3], [4].

\section{VBLAST Scheme Using Soft Symbol Estimate}

We now derive an improved VBLAST scheme by replacing the hard decision in (2) with soft symbol estimate in order to mitigate the effect of error propagation, i.e.,

$$
\mathbf{r}_{i}=\mathbf{r}_{i-1}-\bar{d}_{k_{i-1}}(\mathbf{H})_{k_{i-1}},
$$

where $\bar{d}_{k_{i-1}}$ is the soft estimate of $d_{k_{i-1}}$. In order to further suppress the residual interference in $\mathbf{r}_{i}$, a nulling filter $\mathbf{m}_{i}$ is applied to $\mathbf{r}_{i}$, to obtain

$$
z_{i}=\mathbf{m}_{i}^{\mathcal{H}} \mathbf{r}_{i}
$$

where $\mathbf{m}_{i} \in \mathbb{C}^{M \times 1}$ is chosen by minimizing $\mathrm{E}\left\{\left|\mathbf{m}_{i}^{\mathcal{H}} \mathbf{r}_{i}-d_{k_{i}}\right|^{2}\right\}$ under the MMSE criterion. It can be derived as

$$
\mathbf{m}_{i}=\left[\sum_{j=k_{1}}^{k_{i-1}}(\mathbf{H})_{j}(\mathbf{H})_{j}^{\mathcal{H}} \operatorname{var}\left(d_{k_{j}}\right)+\sum_{j=k_{i}}^{k_{N}}(\mathbf{H})_{j}(\mathbf{H})_{j}^{\mathcal{H}}+\sigma_{u}^{2} \mathbf{I}_{M}\right]^{-1}(\mathbf{H})_{k_{i}},
$$

where

$$
\begin{aligned}
\operatorname{var}\left(d_{k_{j}}\right) & =\mathrm{E}\left[\left|d_{k_{j}}-\bar{d}_{k_{j}}\right|^{2}=\mathrm{E}\left[\left|d_{k_{j}}\right|^{2}\right]-\left|\bar{d}_{k_{j}}\right|^{2} ;\right. \\
\mathrm{E}\left[\left|d_{k_{j}}\right|^{2}\right] & =\sum_{m=0}^{q-1}\left|s_{m}\right|^{2} P_{r}\left(d_{k_{j}}=s_{m}\right) ; \\
\bar{d}_{k_{j}} & =\sum_{m=0}^{q-1} s_{m} P_{r}\left(d_{k_{j}}=s_{m}\right) .
\end{aligned}
$$


In (7), $q$ denotes the modulation level, $s_{m}$ is the $m$ th symbol in the signal constellation. In what follows, we use QPSK and 16-QAM systems as examples to demonstrate how $\bar{d}_{k_{j}}$ can be derived in order to carry out the iterative process.

The nulling filter output can be expressed as $z_{i}=\mathbf{m}_{i}^{\mathcal{H}} \mathbf{r}_{i}=\mu_{i} d_{k_{i}}+\xi_{i}$, where the combined noise and residual interference $\xi_{i}$ can be approximated as a Gaussian random variable [12], [13], i.e., $\xi_{i} \sim \mathcal{C N}\left(0, N_{\xi}\right)$. The parameters $\mu_{i}, N_{\xi}$ can be determined as

$$
\begin{aligned}
\mu_{i} & =\mathrm{E}\left\{z_{i} d_{i}^{*}\right\}=\mathbf{m}_{i}^{\mathcal{H}} \mathrm{E}\left[\mathbf{r}_{i} d_{k_{i}}^{*}\right]=\mathbf{m}_{i}^{\mathcal{H}} \mathbf{C}_{\mathbf{r} d}=\mathbf{m}_{i}^{\mathcal{H}}(\mathbf{H})_{k_{i}} \\
N_{\xi} & =\mathrm{E}\left[\left|\xi_{i}\right|^{2}\right]=\mathrm{E}\left[\left|z_{i}-\mu_{i} d_{i}\right|^{2}\right]=\mathrm{E}\left\{\left|z_{i}\right|^{2}\right\}-\mu_{i}^{2}=\mu_{i}\left(1-\mu_{i}\right)
\end{aligned}
$$

After computing the values of $\mu_{i}$ and $N_{\xi}$, the conditional probability density function (PDF) of the filter output can be obtained as

$$
f\left(z_{i} \mid d_{k_{i}}=s_{m}\right)=\frac{1}{\pi N_{\xi}} \exp (\underbrace{-\frac{\left|z_{i}-\mu_{i} s_{m}\right|^{2}}{N_{\xi}}}_{p\left(s_{m}\right)}),
$$

In QPSK systems, each symbol $d_{k_{i}}$ corresponds to two information bits, denoted as $b_{k_{i}}^{0}$ and $b_{k_{i}}^{1}$. The soft estimate of $d_{k_{i}}$ is computed according to its LLR value as

$$
\bar{d}_{k_{i}}=\tanh \left[\lambda\left(b_{k_{i}}^{0}\right) / 2\right] / \sqrt{2}+j \tanh \left[\lambda\left(b_{k_{i}}^{1}\right) / 2\right] / \sqrt{2},
$$

where $\lambda\left(b_{k_{i}}^{0}\right)$ and $\lambda\left(b_{k_{i}}^{1}\right)$ are the log-likelihood ratio (LLR) for $b_{k_{i}}^{0}$ and $b_{k_{i}}^{1}$, respectively. The former can be computed as

$$
\begin{aligned}
\lambda\left(b_{k_{i}}^{0}\right) & =\ln \frac{f\left(z_{i} \mid b_{k_{i}}^{0}=1\right)}{f\left(z_{i} \mid b_{k_{i}}^{0}=0\right)}=\ln \frac{f\left(z_{i} \mid d_{k_{i}}=s_{3}\right)+f\left(z_{i} \mid d_{k_{i}}=s_{4}\right)}{f\left(z_{i} \mid d_{k_{i}}=s_{1}\right)+f\left(z_{i} \mid d_{k_{i}}=s_{2}\right)} \\
& \approx \ln \frac{\exp \left(-\left|z_{i}-\mu_{i} d_{k_{i}}^{+}\right|^{2} / N_{\xi}\right)}{\exp \left(-\left|z_{i}-\mu_{i} d_{k_{i}}^{-}\right|^{2} / N_{\xi}\right)}=\frac{1}{N_{\xi}}\left\{\left|z_{i}-\mu_{i} d_{k_{i}}^{-}\right|^{2}-\left|z_{i}-\mu_{i} d_{k_{i}}^{+}\right|^{2}\right\} \\
& =\frac{2}{1-\mu_{i}} \operatorname{Re}\left\{d_{k_{i}}^{+*} z_{i}-d_{k_{i}}^{-*} z_{i}\right\}
\end{aligned}
$$

where $d_{k_{i}}^{+}$denotes the QPSK symbol corresponding to $\max \left\{p\left(s_{3}\right), p\left(s_{4}\right)\right\}$, and $d_{k_{i}}^{-}$denotes the QPSK symbol corresponding to $\max \left\{p\left(s_{1}\right), p\left(s_{2}\right)\right\}$, since the real part of the symbols $s_{3}, s_{4}$ corresponds to 1 , and the real part of the symbols $s_{1}, s_{2}$ corresponds to 0 as shown in Fig. 1.

The LLR value for the second information bit $b_{k_{i}}^{1}$ can be obtained in a similar manner.

For the 16-QAM constellation shown in Fig. 2, each symbol $d_{k_{i}}$ is associated with four bits $b_{k_{i}}^{0}, b_{k_{i}}^{1}, b_{k_{i}}^{2}, b_{k_{i}}^{3}$, their LLR values of can be obtained as

$$
\begin{aligned}
& \lambda\left(b_{k_{i}}^{0}\right)=\ln \frac{f\left(z_{i} \mid b_{k_{i}}^{0}=1\right)}{f\left(z_{i} \mid b_{k_{i}}^{0}=0\right)}=\ln \frac{\sum_{s \in\left(c_{2}, c_{3}\right)} f\left(z_{i} \mid d_{k_{i}}\right)}{\sum_{s \in\left(c_{0}, c_{1}\right)} f\left(z_{i} \mid d_{k_{i}}\right)} ; \quad \lambda\left(b_{k_{i}}^{1}\right)=\ln \frac{f\left(z_{i} \mid b_{k_{i}}^{1}=1\right)}{f\left(z_{i} \mid b_{k_{i}}^{1}=0\right)}=\ln \frac{\sum_{s \in\left(c_{0}, c_{3}\right)} f\left(z_{i} \mid d_{k_{i}}\right)}{\sum_{s \in\left(c_{1}, c_{2}\right)} f\left(z_{i} \mid d_{k_{i}}\right)} ; \\
& \lambda\left(b_{k_{i}}^{2}\right)=\ln \frac{f\left(z_{i} \mid b_{k_{i}}^{2}=1\right)}{f\left(z_{i} \mid b_{k_{i}}^{2}=0\right)}=\ln \frac{\sum_{s \in\left(r_{0}, r_{1}\right)} f\left(z_{i} \mid d_{k_{i}}\right)}{\sum_{s \in\left(r_{2}, r_{3}\right)} f\left(z_{i} \mid d_{k_{i}}\right)} ; \quad \lambda\left(b_{k_{i}}^{3}\right)=\ln \frac{f\left(z_{i} \mid b_{k_{i}}^{3}=1\right)}{f\left(z_{i} \mid b_{k_{i}}^{3}=0\right)}=\ln \frac{\sum_{s \in\left(r_{0}, r_{3}\right)} f\left(z_{i} \mid d_{k_{i}}\right)}{\sum_{s \in\left(r_{1}, r_{2}\right)} f\left(z_{i} \mid d_{k_{i}}\right)},
\end{aligned}
$$




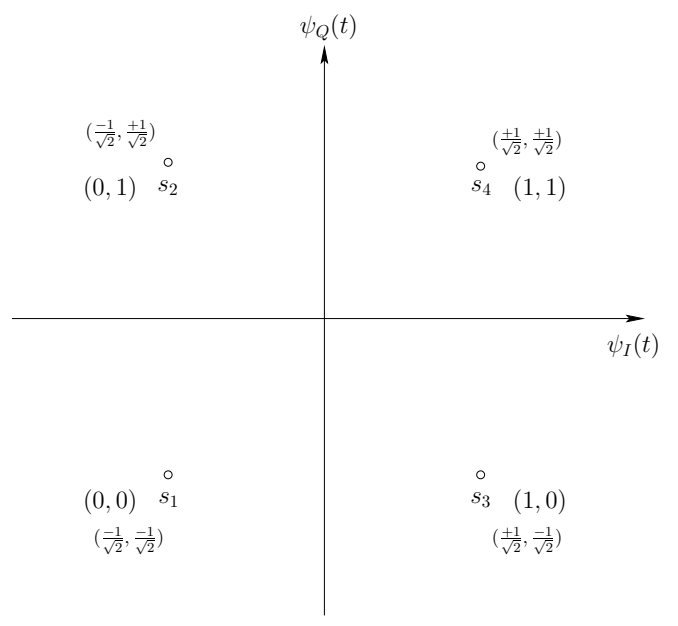

Fig. 1. QPSK constellation and bit-to-symbol mapping.

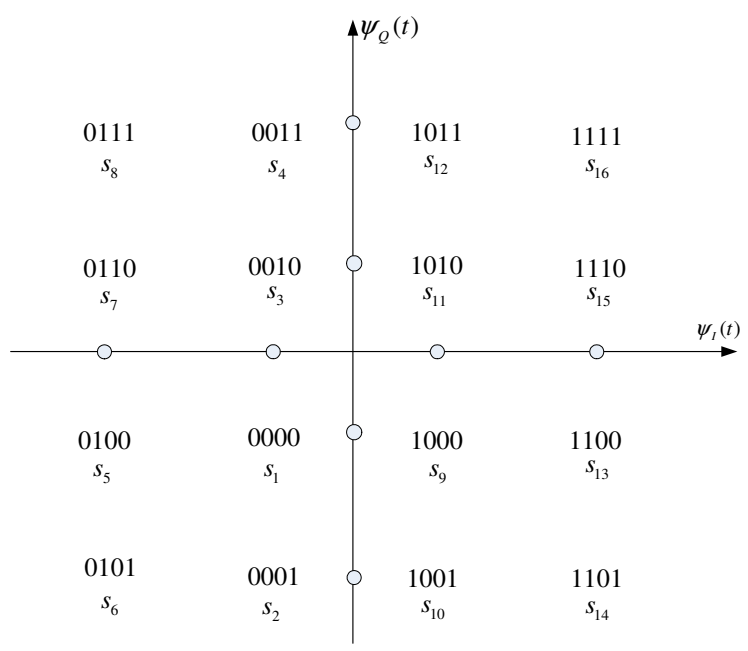

Fig. 2. 16-QAM constellation and bit-to-symbol mapping.

where $s \in\left(r_{i}\right), s \in\left(c_{j}\right)$ denotes the symbols that lie in the $i^{t h}$ row and the $j^{\text {th }}$ column of the signal constellation, respectively. The above equations hold due to the symbol-to-bit mapping shown in Fig. 2. For example, $f\left(z_{i} \mid b_{k_{i}}^{0}=\right.$ $0)=\sum_{s \in\left(c_{0}, c_{1}\right)} f\left(z_{i} \mid d_{k_{i}}\right)$ since the first bit is 0 for all the symbols located at the first and second columns of the constellation.

Utilizing the fact that one term usually dominates each sum, $\lambda\left(b_{k_{i}}^{0} ; O\right)$ can be approximated as

$$
\begin{aligned}
& \lambda\left(b_{k_{i}}^{0} ; O\right) \approx \ln \frac{\exp \left(-\left|z_{i}-\mu_{i} s_{+}\right|^{2} / N_{\xi}\right)}{\exp \left(-\left|z_{i}-\mu_{i} s_{-}\right|^{2} / N_{\xi}\right)}=\frac{1}{N_{\xi}}\left\{\left|z_{i}-\mu_{i} s_{-}\right|^{2}-\left|z_{i}-\mu_{i} s_{+}\right|^{2}\right\} \\
& =\frac{1}{\mu_{i}} \operatorname{Re}\left\{\left[2\left(s_{+}\right)^{*} z_{i}-\mu_{i}\left|d_{+}\right|^{2}\right]-\left[2\left(d_{-}\right)^{*} z_{i}-\mu_{i}\left|d_{-}\right|^{2}\right]\right\}
\end{aligned}
$$


where $s_{+}$denotes the symbol corresponding to

$$
\max \left\{p\left(s_{9}\right), p\left(s_{10}\right), p\left(s_{11}\right), p\left(s_{12}\right), p\left(s_{13}\right), p\left(s_{14}\right), p\left(s_{15}\right), p\left(s_{16}\right)\right\},
$$

and $s_{-}$denotes the symbol corresponding to

$$
\max \left\{p\left(s_{1}\right), p\left(s_{2}\right), p\left(s_{3}\right), p\left(s_{4}\right), p\left(s_{5}\right), p\left(s_{6}\right), p\left(s_{7}\right), p\left(s_{8}\right)\right\} \text {. }
$$

The other LLR values $\lambda\left(b_{k_{i}}^{1}\right), \lambda\left(b_{k_{i}}^{2}\right), \lambda\left(b_{k_{i}}^{3}\right)$ can be derived similarly. Note that a further approximation method for deriving soft output was proposed for square M-QAM constellation in [11].

According to the symbol-to-bit mapping shown in Fig. 2, we have

$$
\begin{gathered}
P_{r}\left(d_{k_{i}}=s_{1}\right)=P_{r}\left(b_{k_{i}}^{0}=0\right) \cdot P_{r}\left(b_{k_{i}}^{1}=0\right) \cdot P_{r}\left(b_{k_{i}}^{2}=0\right) \cdot P_{r}\left(b_{k_{i}}^{3}=0\right) \\
P_{r}\left(d_{k_{i}}=s_{2}\right)=P_{r}\left(b_{k_{i}}^{0}=0\right) \cdot P_{r}\left(b_{k_{i}}^{1}=0\right) \cdot P_{r}\left(b_{k_{i}}^{2}=0\right) \cdot P_{r}\left(b_{k_{i}}^{3}=1\right) \\
\vdots \\
P_{r}\left(d_{k_{i}}=s_{16}\right)=P_{r}\left(b_{k_{i}}^{0}=1\right) \cdot P_{r}\left(b_{k_{i}}^{1}=1\right) \cdot P_{r}\left(b_{k_{i}}^{2}=1\right) \cdot P_{r}\left(b_{k_{i}}^{3}=1\right),
\end{gathered}
$$

where

$$
P_{r}\left(b_{k_{i}}^{p}=1\right)=\frac{e^{\lambda\left(b_{k_{i}}^{p}\right)}}{1+e^{\lambda\left(b_{k_{i}}^{p}\right)}} ; \quad P_{r}\left(b_{k_{i}}^{p}=0\right)=\frac{1}{1+e^{\lambda\left(b_{k_{i}}^{p}\right)}} .
$$

With the a priori probability of each symbol $P_{r}\left(d_{k_{i}}=s_{j}\right)$, the soft estimate $\bar{d}_{k_{i}}$ in (4) can be calculated by (7).

In what follows, we show an alternative implementation of the VBLAST algorithm utilizing soft symbol estimate but without nulling filter. Recall that Eq. (4) can be expanded as

$$
\mathbf{r}_{i}=d_{k_{i}}(\mathbf{H})_{i}+\underbrace{\sum_{j=k_{1}}^{k_{i}-1}\left(d_{k_{j}}-\bar{d}_{k_{j}}\right)(\mathbf{H})_{j}+\sum_{j=k_{i+1}}^{k_{N}} d_{k_{j}}(\mathbf{H})_{j}+\mathbf{u}}_{\mathbf{w}_{i}}
$$

where the first term is the desired signal, the last three terms are the combined interference and noise denoted by $\mathbf{w}_{i}$. The elements of $\mathbf{w}_{i}$ are assumed to be zero mean Gaussian random variables. Let $\mathbf{M}_{i}$ denote the covariance matrix of $\mathbf{w}_{i}$, it can be easily shown that

$$
\mathbf{M}_{i}=\sum_{j=k_{1}}^{k_{i-1}}(\mathbf{H})_{j}(\mathbf{H})_{j}^{\mathcal{H}} \operatorname{var}\left(d_{k_{j}}\right)+\sum_{j=k_{i+1}}^{k_{N}}(\mathbf{H})_{j}(\mathbf{H})_{j}^{\mathcal{H}}+\sigma_{u}^{2} \mathbf{I}_{M} .
$$

The conditional PDF of $\mathbf{r}_{i}$ can thus be derived as

$$
f\left(\mathbf{r}_{i} \mid d_{k_{i}}=s_{m}\right)=\frac{1}{2 \pi \sqrt{\operatorname{det} \mathbf{M}_{i}}} \exp (\underbrace{-\frac{1}{2}\left(\mathbf{r}_{i}-s_{m}(\mathbf{H})_{i}\right)^{\mathcal{H}} \mathbf{M}_{i}^{-1}\left(\mathbf{r}_{i}-s_{m}(\mathbf{H})_{i}\right)}_{g\left(s_{m}\right)}) .
$$

In the case of QPSK modulation, the LLR value of $b_{k_{i}}^{0}$ can be computed as

$$
\begin{aligned}
\lambda\left(b_{k_{i}}^{0}\right) & =\ln \frac{f\left(\mathbf{r}_{i} \mid b_{k_{i}}^{0}=1\right)}{f\left(\mathbf{r}_{i} \mid b_{k_{i}}^{0}=1\right)} \approx \ln \frac{\exp \left[-\frac{1}{2}\left(\mathbf{r}_{i}-s_{+}(\mathbf{H})_{i}\right)^{\mathcal{H}} \mathbf{M}_{i}^{-1}\left(\mathbf{r}_{i}-s_{+}(\mathbf{H})_{i}\right)\right]}{\exp \left[-\frac{1}{2}\left(\mathbf{r}_{i}-s_{-}(\mathbf{H})_{i}\right)^{\mathcal{H}} \mathbf{M}_{i}^{-1}\left(\mathbf{r}_{i}-s_{-}(\mathbf{H})_{i}\right)\right]} \\
& =\frac{1}{2}\left(\mathbf{r}_{i}-s_{-}(\mathbf{H})_{i}\right)^{\mathcal{H}} \mathbf{M}_{i}^{-1}\left(\mathbf{r}_{i}-s_{-}(\mathbf{H})_{i}\right)-\frac{1}{2}\left(\mathbf{r}_{i}-s_{+}(\mathbf{H})_{i}\right)^{\mathcal{H}} \mathbf{M}_{i}^{-1}\left(\mathbf{r}_{i}-s_{+}(\mathbf{H})_{i}\right),
\end{aligned}
$$


where $s_{+}$denotes the symbol $d_{k_{i}}$ corresponding to $\max \left\{g\left(s_{3}\right), g\left(s_{4}\right)\right\}$; while $s_{-}$denotes the symbol $d_{k_{i}}$ corresponding to $\max \left\{g\left(s_{1}\right), g\left(s_{2}\right)\right\}$. The LLR value for the second information bit $b_{k_{i}}^{1}$ in the QPSK system as well as LLRs for the 16QAM system can be obtained in a similar manner.

\section{VBLAST Scheme Utilizing Complete Second-Order Statistics}

For a complex random vector $\mathbf{r}_{i}$, its second-order statistics are completely characterized by its autocorrelation matrix $\mathbf{C}_{\mathbf{r r}}=\mathrm{E}\left[\mathbf{r}_{i} \mathbf{r}_{i}^{\mathcal{H}}\right]$ as well as its pseudo-autocorrelation matrix $\tilde{\mathbf{C}}_{\mathbf{r r}}=\mathrm{E}\left[\mathbf{r}_{i} \mathbf{r}_{i}^{\mathcal{T}}\right]$. Most existing receiver algorithms only use the information contained in the autocorrelation function of the observed signal. The pseudoautocorrelation matrix $\tilde{\mathbf{C}}_{\mathbf{r r}}$ is usually not considered and is implicitly assumed to be zero. While this is the optimum strategy when dealing with circular complex random processes, i.e., when $\tilde{\mathbf{C}}_{\mathbf{r r}}=\mathbf{0}$, it turns out to be sub-optimum in situations where the transmitted signals and/or interference are noncircular random processes, i.e., when $\tilde{\mathbf{C}}_{\mathbf{r r}} \neq \mathbf{0}$. As we demonstrated in [14], the condition of the signal subspace can be improved by exploiting the information contained in the pseudo-autocorrelation matrix. It was also shown in [15] that the performance of the blind multiuser detectors can be improved by utilizing $\tilde{\mathbf{C}}_{\mathbf{r r}}$ in the presence of noncircular narrow-band interference (NBI).

In the sequel, we show how $\tilde{\mathbf{C}}_{\mathrm{rr}}$ can be incorporated into the VBLAST detection scheme to optimize the system performance. To this end, let us define

$$
z_{i}=\boldsymbol{w}_{i}^{\mathcal{H}} \mathbf{y}_{i}
$$

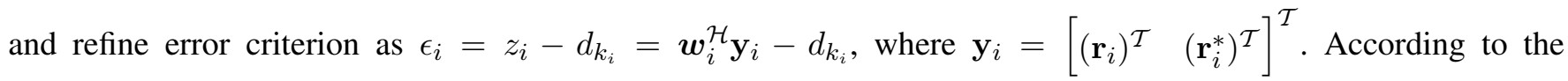
orthogonality principle [16], the mean-square value of the estimation error $\epsilon_{i}$ is only minimal, if it is orthogonal to the observation vector $\mathbf{y}_{i}$, i.e.,

$$
\mathrm{E}\left[\mathbf{y}_{i} \epsilon_{i}^{*}\right]=\mathrm{E}\left[\mathbf{y}_{i}\left(\boldsymbol{w}_{i}^{\mathcal{H}} \mathbf{y}_{i}-d_{k_{i}}\right)^{\mathcal{H}}\right]=\mathbf{0}
$$

leading to the solution for the new nulling fiter

$$
\boldsymbol{w}_{i}=\mathbf{C}_{\mathbf{y y}}^{-1} \mathbf{C}_{\mathbf{y} d}
$$

where

$$
\begin{aligned}
& \mathbf{C}_{\mathbf{y y}}=\mathrm{E}\left\{\mathbf{y}_{i} \mathbf{y}_{i}^{\mathcal{H}}\right\}=\mathrm{E}\left\{\left[\begin{array}{l}
\mathbf{r}_{i} \\
\mathbf{r}_{i}^{*}
\end{array}\right]\left[\begin{array}{ll}
\mathbf{r}_{i}^{\mathcal{H}} & \mathbf{r}_{i}^{\mathcal{T}}
\end{array}\right]\right\}=\left[\begin{array}{cc}
\mathbf{C}_{\mathbf{r r}} & \tilde{\mathbf{C}}_{\mathbf{r r}} \\
\tilde{\mathbf{C}}_{\mathbf{r r}}^{*} & \mathbf{C}_{\mathbf{r r}}^{*}
\end{array}\right], \\
& \mathbf{C}_{\mathbf{y} d}=\mathrm{E}\left\{\mathbf{y}_{i} d_{k_{i}}^{*}\right\}=\mathrm{E}\left\{\left[\begin{array}{l}
\mathbf{r}_{i} \\
\mathbf{r}_{i}^{*}
\end{array}\right] d_{k_{i}}^{*}\right\}=\left[\begin{array}{c}
\mathbf{C}_{\mathbf{r} d} \\
\tilde{\mathbf{C}}_{\mathbf{r} d}^{*}
\end{array}\right]=\left[\begin{array}{c}
(\mathbf{H})_{k_{i}} \\
\mathbf{0}
\end{array}\right] .
\end{aligned}
$$


The matrix $\tilde{\mathbf{C}}_{\mathbf{r} d}^{*}=\mathbf{0}$ since $\mathrm{E}\left[\left(d_{k_{i}}-\bar{d}_{k_{i}}\right)^{*} d_{k_{n}}^{*}\right]=0$ if $k_{i} \neq k_{n}$; and $\mathrm{E}\left[d_{k_{i}}^{*} d_{k_{i}}^{*}\right]=0$ for complex signal constellations. The autocorrelation matrix $\mathbf{C}_{\mathbf{r r}}$ and the pseudo-autocorrelation matrix $\tilde{\mathbf{C}}_{\mathbf{r r}}$ can be computed as

$$
\begin{aligned}
& \mathbf{C}_{\mathbf{r r}}=\sum_{j=k_{1}}^{k_{i-1}}(\mathbf{H})_{j}(\mathbf{H})_{j}^{\mathcal{H}} \operatorname{var}\left(d_{k_{j}}\right)+\sum_{j=k_{i}}^{k_{N}}(\mathbf{H})_{j}(\mathbf{H})_{j}^{\mathcal{H}}+\sigma_{u}^{2} \mathbf{I}_{M} ; \\
& \tilde{\mathbf{C}}_{\mathbf{r r}}=\sum_{j=k_{1}}^{k_{i-1}}(\mathbf{H})_{j}(\mathbf{H})_{j}^{\mathcal{T}} L_{k_{j}},
\end{aligned}
$$

where

$$
L_{k_{j}}=\mathrm{E}\left[\left(d_{k_{j}}-\bar{d}_{k_{j}}\right)^{2}\right]=\mathrm{E}\left[d_{k_{j}, I}^{2}\right]-\mathrm{E}\left[d_{k_{j}, Q}^{2}\right]+\bar{d}_{k_{j}, Q}^{2}-\bar{d}_{k_{j}, I}^{2}
$$

and

$$
\begin{aligned}
\mathrm{E}\left[d_{k_{i}, I}^{2}\right] & =\sum_{m=0}^{q-1} s_{m, I}^{2} P_{r}\left(d_{k_{i}}=s_{m}\right) \\
\mathrm{E}\left[d_{k_{i}, Q}^{2}\right] & =\sum_{m=0}^{q-1} s_{m, Q}^{2} P_{r}\left(d_{k_{i}}=s_{m}\right) .
\end{aligned}
$$

In (20) and (21), $d_{k_{j}, I}, d_{k_{j}, Q}$ are the real and imaginary parts of $d_{k_{j}} ; s_{m, I} s_{m, Q}$ are the real and imaginary parts of $s_{m}$, respectively. Let us denote a complex symbol $x=x_{I}+j x_{Q}$, and $\bar{x}=\bar{x}_{I}+j \bar{x}_{Q}$, where $\bar{x}=\mathrm{E}[x]$. Eq. (20) holds since

$$
\begin{aligned}
\mathrm{E}\left[(x-\bar{x})^{2}\right] & =\mathrm{E}\left[x^{2}\right]-\bar{x}^{2}=\mathrm{E}\left[x_{I}^{2}+2 j x_{I} x_{Q}-x_{Q}^{2}\right]-\bar{x}_{I}^{2}-2 j \bar{x}_{I} \bar{x}_{Q}+\bar{x}_{Q}^{2} \\
& =\mathrm{E}\left[x_{I}^{2}\right]-\mathrm{E}\left[x_{Q}^{2}\right]+\bar{x}_{Q}^{2}-\bar{x}_{I}^{2} .
\end{aligned}
$$

Note that for the VBLAST detector introduced in the previous section, the nulling filter $\mathbf{m}_{i}$ in (6) is calculated using only the autocorrelation matrix $\mathbf{C}_{\mathbf{r r}}=\mathrm{E}\left[\mathbf{r}_{i} \mathbf{r}_{i}^{\mathcal{H}}\right]=\sum_{j=k_{1}}^{k_{i-1}}(\mathbf{H})_{j}(\mathbf{H})_{j}^{\mathcal{H}} \operatorname{var}\left(d_{k_{j}}\right)+\sum_{j=k_{i}}^{k_{N}}(\mathbf{H})_{j}(\mathbf{H})_{j}^{\mathcal{H}}+\sigma_{u}^{2} \mathbf{I}_{M}$, i.e., $\mathbf{m}_{i}=\mathbf{C}_{\mathbf{r r}}^{-1} \mathbf{C}_{\mathbf{r} d}$, leading to the sub-optimum solution, which will be verified by the simulation results shown in Section V.

The refined nulling filter output can be expressed as $z_{i}=\boldsymbol{w}_{i}^{\mathcal{H}} \mathbf{y}_{i}=\mu_{i} d_{k_{i}}+\nu_{i} d_{k_{i}}^{*}+\eta_{i}$, where the combined noise and residual interference $\eta_{i}$ can be approximated as a Gaussian random variable. Next, we derive the LLR values for QPSK and 16-QAM systems based on the assumption that the interference-plus-noise term $\eta_{i}$ at the output of the nulling filter is also a noncircular random process. The parameters $\mu_{i}, \nu_{i}, N_{\eta}$ can be computed as

$$
\begin{aligned}
\mu_{i} & =\mathrm{E}\left\{z_{i} d_{k_{i}}^{*}\right\}=\boldsymbol{w}_{i}^{\mathcal{H}} \mathrm{E}\left[\mathbf{y}_{i} d_{k_{i}}^{*}\right]=\boldsymbol{w}_{i}^{\mathcal{H}} \mathbf{C}_{\mathbf{y} d}=\boldsymbol{w}_{i}^{\mathcal{H}}\left[\begin{array}{c}
(\mathbf{H})_{k_{i}} \\
\mathbf{0}
\end{array}\right] \\
\nu_{i} & =\mathrm{E}\left\{z_{i} d_{k_{i}}\right\}=\boldsymbol{w}_{i}^{\mathcal{H}} \mathrm{E}\left[\mathbf{y}_{i} d_{k_{i}}\right]=\boldsymbol{w}_{i}^{\mathcal{H}} \tilde{\mathbf{C}}_{\mathbf{y} d}=\boldsymbol{w}_{i}^{\mathcal{H}}\left[\begin{array}{c}
\mathbf{0} \\
(\mathbf{H})_{k_{i}}^{*}
\end{array}\right] \\
N_{\eta} & =\mathrm{E}\left[\left|\eta_{i}\right|^{2}\right]=\mathrm{E}\left[\left|z_{i}-\mu_{i} d_{k_{i}}-\nu_{i} d_{k_{i}}^{*}\right|^{2}\right] \\
& =\mathrm{E}\left\{\left|z_{i}\right|^{2}\right\}-\left|\mu_{i}\right|^{2}-\left|\nu_{i}\right|^{2}=\mu_{i}^{*}-\left|\mu_{i}\right|^{2}-\left|\nu_{i}\right|^{2} .
\end{aligned}
$$


The above equation holds since $z_{i}=\boldsymbol{w}_{i}^{\mathcal{H}} \mathbf{y}_{i}$ and $\boldsymbol{w}_{i}=\mathbf{C}_{\mathbf{y y}}^{-1} \mathbf{C}_{\mathbf{y} d}$. Therefore,

$$
\mathrm{E}\left\{\left|z_{i}\right|^{2}\right\}=\mathrm{E}\left\{\boldsymbol{w}_{i}^{\mathcal{H}} \mathbf{y}_{i} \mathbf{y}_{i}^{\mathcal{H}} \boldsymbol{w}_{i}\right\}=\boldsymbol{w}_{i}^{\mathcal{H}} \mathbf{C}_{\mathbf{y y}} \boldsymbol{w}_{i}=\mathbf{C}_{\mathbf{y} d}^{\mathcal{H}} \mathbf{C}_{\mathbf{y y}}^{-1} \mathbf{C}_{\mathbf{y y}} \boldsymbol{w}_{i}=\mathbf{C}_{\mathbf{y} d}^{\mathcal{H}} \boldsymbol{w}_{i}=\mu_{i}^{*} .
$$

In the derivation of the proposed scheme, we take into account the noncircular nature of $\eta_{i}$, and utilize the fact that $\tilde{N}_{\eta}=\mathrm{E}\left[\eta_{i}^{2}\right] \neq 0$, which can be computed as

$$
\begin{aligned}
\tilde{N}_{\eta} & =\mathrm{E}\left[\eta^{2}\right]=\mathrm{E}\left[\left(z_{i}-\mu_{i} d_{k_{i}}-\nu_{i} d_{k_{i}}^{*}\right)^{2}\right]=\mathrm{E}\left[\left(z_{i}-\mu_{i} d_{k_{i}}-\nu_{i} d_{k_{i}}^{*}\right)\left(z_{i}-\mu_{i} d_{k_{i}}-\nu_{i} d_{k_{i}}^{*}\right)\right] \\
& =\mathrm{E}\left\{z_{i}^{2}\right\}-2 \mu_{i} \nu_{i}=\mathrm{E}\left\{\boldsymbol{w}_{i}^{\mathcal{H}} \mathbf{y}_{i} \mathbf{y}_{i}^{\mathcal{T}} \boldsymbol{w}_{i}^{*}\right\}-2 \mu_{i} \nu_{i}=\boldsymbol{w}_{i}^{\mathcal{H}} \tilde{\mathbf{C}}_{\mathbf{y y}} \boldsymbol{w}_{i}^{*}-2 \mu_{i} \nu_{i} .
\end{aligned}
$$

Eq. (23) follows from the fact that $\boldsymbol{w}_{i}^{\mathcal{H}} \mathbf{y}=\mathbf{y}_{i}^{\mathcal{T}} \boldsymbol{w}_{i}^{*}$, and

$$
\mathrm{E}\left\{z_{i}^{2}\right\}=\mathrm{E}\left\{\boldsymbol{w}_{i}^{\mathcal{H}} \mathbf{y}_{i} \mathbf{y}_{i}^{\mathcal{T}} \boldsymbol{w}_{i}^{*}\right\}=\boldsymbol{w}_{i}^{\mathcal{H}} \mathrm{E}\left\{\mathbf{y}_{i} \mathbf{y}_{i}^{\mathcal{T}}\right\} \boldsymbol{w}_{i}^{*}=\boldsymbol{w}_{i}^{\mathcal{H}} \tilde{\mathbf{C}}_{\mathbf{y y}} \boldsymbol{w}_{i}^{*},
$$

where

$$
\tilde{\mathbf{C}}_{\mathbf{y y}}=\mathrm{E}\left\{\mathbf{y}_{i} \mathbf{y}_{i}^{\mathcal{T}}\right\}=\mathrm{E}\left\{\left[\begin{array}{c}
\mathbf{r}_{i} \\
\mathbf{r}_{i}^{*}
\end{array}\right]\left[\begin{array}{ll}
\mathbf{r}_{i}^{\mathcal{T}} & \mathbf{r}_{i}^{\mathcal{H}}
\end{array}\right]\right\}=\left[\begin{array}{ll}
\tilde{\mathbf{C}}_{\mathbf{r r}} & \mathbf{C}_{\mathbf{r r}} \\
\mathbf{C}_{\mathbf{r r}}^{*} & \tilde{\mathbf{C}}_{\mathbf{r r}}^{*}
\end{array}\right] \text {. }
$$

Let us denote $z_{i}=z_{i, I}+j z_{i, Q}, \mu_{i}=\mu_{i, I}+j \mu_{i, Q}, \nu_{i}=\nu_{i, I}+j \nu_{i, Q}, d_{k_{i}}=d_{k_{i}, I}+j d_{k_{i}, Q}$, and $\eta_{i}=\eta_{i, I}+j \eta_{i, Q}$. The filter output $z_{i}=\mu_{i} d_{k_{i}}+\nu_{i} d_{k_{i}}^{*}+\eta_{i}$ can be reformed as

$$
\underbrace{\left[\begin{array}{c}
z_{i, I} \\
z_{i, Q}
\end{array}\right]}_{\mathbf{z}_{i}}=\underbrace{\left[\begin{array}{l}
\left(\mu_{i}+\nu_{i, I}\right) d_{i_{i}, I}+\nu_{i, Q} d_{k_{i}, Q} \\
\left(\mu_{i}-\nu_{i, I}\right) d_{k_{i}, Q}+\nu_{i, Q} d_{k_{i}, I}
\end{array}\right]}_{\mathbf{d}_{k_{i}}}+\underbrace{\left[\begin{array}{c}
\eta_{i, I} \\
\eta_{i, Q}
\end{array}\right]}_{\boldsymbol{\eta}_{i}}
$$

Since the probability distribution of a complex random variable or vector is a joint distribution of its real and imaginary part, we have

$$
f\left(z_{i} \mid d_{k_{i}}=s_{m}\right)=f\left(\mathbf{z}_{i} \mid \mathbf{d}_{k_{i}}=s_{m}\right)=\frac{1}{2 \pi \sqrt{\operatorname{det} \boldsymbol{\Sigma}_{i}}} \exp (\underbrace{-\frac{1}{2}\left(\mathbf{z}_{i}-\mathbf{d}_{k_{i}}\right)^{\mathcal{H}} \boldsymbol{\Sigma}_{i}^{-1}\left(\mathbf{z}_{k_{i}}-\mathbf{d}_{k_{i}}\right)}_{h\left(s_{m}\right)})
$$

where $\mathbf{d}_{k_{i}}$ is formed according to (24) given that $d_{k_{i}}=s_{m}$, and the covariance matrix of the Gaussian noise is $\boldsymbol{\Sigma}_{i}=\mathrm{E}\left[\boldsymbol{\eta}_{i} \boldsymbol{\eta}_{i}^{\mathcal{H}}\right]$. Define the mapping matrix as $\mathbf{J}=\frac{1}{\sqrt{2}}\left[\begin{array}{cc}1 & j \\ 1 & -j\end{array}\right]$, which is an unitary matrix since $\mathbf{J} \mathbf{J}^{\mathcal{H}}=\mathbf{J}^{\mathcal{H}} \mathbf{J}=\mathbf{I}$, and $\mathbf{J}^{-1}=\mathbf{J}^{\mathcal{H}}$. We have

$$
\mathbf{J} \boldsymbol{\Sigma}_{i} \mathbf{J}^{\mathcal{H}}=\mathbf{J} \mathrm{E}\left[\boldsymbol{\eta}_{i} \boldsymbol{\eta}_{i}^{\mathcal{H}}\right] \mathbf{J}^{\mathcal{H}}=\mathrm{E}\left[\left(\mathbf{J} \boldsymbol{\eta}_{i}\right)\left(\mathbf{J} \boldsymbol{\eta}_{i}\right)^{\mathcal{H}}\right]=\frac{1}{2} \mathrm{E}\left[\boldsymbol{\epsilon}_{i} \boldsymbol{\epsilon}_{i}^{\mathcal{H}}\right]=\frac{1}{2} \boldsymbol{\Phi}_{i},
$$

where $\boldsymbol{\epsilon}_{i}=\left[\begin{array}{c}\eta_{i} \\ \eta_{i}^{*}\end{array}\right]$, and

$$
\boldsymbol{\Phi}_{i}=\mathrm{E}\left[\boldsymbol{\epsilon}_{i} \boldsymbol{\epsilon}_{i}^{\mathcal{H}}\right]=\mathrm{E}\left\{\left[\begin{array}{c}
\eta_{i} \\
\eta_{i}^{*}
\end{array}\right]\left[\begin{array}{cc}
\eta_{i}^{*} & \eta_{i}
\end{array}\right]\right\}=\mathrm{E}\left\{\left[\begin{array}{cc}
\eta_{i} \eta_{i}^{*} & \eta_{i} \eta_{i} \\
\eta_{i}^{*} \eta_{i}^{*} & \eta_{i}^{*} \eta_{i}
\end{array}\right]\right\}=\left[\begin{array}{cc}
N_{\eta} & \tilde{N}_{\eta} \\
\tilde{N}_{\eta}^{*} & N_{\eta}
\end{array}\right]
$$


COMPLEXITY FOR ONE SYMBOL ESTIMATE FOR THE ALGORITHMS CONSIDERED.

\begin{tabular}{|c|c|c|c|c|}
\hline \hline operations & $\div$ & $\times$ & $+/-$ & $\tanh$ \\
\hline VBLAST-I & $2 N^{2}$ & $4 N^{3}+2 N^{2}+N$ & $4 N^{3}$ & 0 \\
\hline VBLAST-II & $4 N^{2}$ & $6 N^{3}+2 N^{2}+7 N$ & $6 N^{3}$ & 2 \\
\hline VBLAST-III & $10 N^{2}+4$ & $20 N^{3}+10 N^{2}+11 N+70$ & $20 N^{3}+8 N^{2}+3 N+47$ & 2 \\
\hline
\end{tabular}

From (26), we have $\boldsymbol{\Sigma}_{i}=\frac{1}{2} \mathbf{J}^{\mathcal{H}} \boldsymbol{\Phi}_{i} \mathbf{J}$, and $\boldsymbol{\Sigma}_{i}^{-1}=2 \mathbf{J}^{\mathcal{H}} \boldsymbol{\Phi}_{n}^{-1} \mathbf{J}$. The PDF in (25) can thus be reformed as

$$
f\left(z_{i} \mid d_{k_{i}}\right)=\frac{1}{2 \pi \sqrt{\operatorname{det} \mathbf{\Sigma}_{i}}} \exp \left[-\left(\mathbf{z}_{i}-\mathbf{d}_{k_{i}}\right)^{\mathcal{H}} \mathbf{J}^{\mathcal{H}} \boldsymbol{\Phi}_{i}^{-1} \mathbf{J}\left(\mathbf{z}_{i}-\mathbf{d}_{k_{i}}\right)\right]
$$

In the case of QPSK modulation, the LLR value of $b_{k_{i}}^{0}$ can be computed as

$$
\begin{aligned}
\lambda\left(b_{k_{i}}^{0}\right) & =\ln \frac{f\left(z_{i} \mid b_{k_{i}}^{0}=1\right)}{f\left(z_{i} \mid b_{k_{i}}^{0}=0\right)}=\ln \frac{f\left(z_{i} \mid d_{k_{i}, I}=+1 / \sqrt{2}\right)}{f\left(z_{i} \mid d_{k_{i}, I}=-1 / \sqrt{2}\right)} \approx \ln \frac{\exp \left[-\left(\mathbf{z}_{i}-\mathbf{d}_{+}\right)^{\mathcal{H}} \mathbf{J}^{\mathcal{H}} \boldsymbol{\Phi}_{i}^{-1} \mathbf{J}\left(\mathbf{z}_{i}-\mathbf{d}_{+}\right)\right]}{\exp \left[-\left(\mathbf{z}_{i}-\mathbf{d}_{-}\right)^{\mathcal{H}} \mathbf{J}^{\mathcal{H}} \mathbf{\Phi}_{i}^{-1} \mathbf{J}\left(\mathbf{z}_{i}-\mathbf{d}_{-}\right)\right]} \\
& =\left(\mathbf{z}_{i}-\mathbf{d}_{-}\right)^{\mathcal{H}} \mathbf{J}^{\mathcal{H}} \boldsymbol{\Phi}_{i}^{-1} \mathbf{J}\left(\mathbf{z}_{i}-\mathbf{d}_{-}\right)-\left(\mathbf{z}_{i}-\mathbf{d}_{+}\right)^{\mathcal{H}} \mathbf{J}^{\mathcal{H}} \boldsymbol{\Phi}_{i}^{-1} \mathbf{J}\left(\mathbf{z}_{i}-\mathbf{d}_{+}\right),
\end{aligned}
$$

where $\mathbf{d}_{+}$denotes the vector $\mathbf{d}_{k_{i}}$ corresponding to $\max \left\{h\left(s_{3}\right), h\left(s_{4}\right)\right\}$ and $\mathbf{d}_{-}$denotes the vector $\mathbf{d}_{k_{i}}$ corresponding to $\max \left\{h\left(s_{1}\right), h\left(s_{2}\right)\right\}$.

The LLR value for $\lambda\left(b_{k_{i}}^{1}\right)$ can be derived similarly. Then we use Equ. (9) to convert LLRs to soft symbol estimate $\bar{d}_{k_{i}}$, which is needed for the interference cancellation at the next iteration.

In the LLR calculation for the bit $b_{k_{i}}^{0}$ in the 16-QAM system, $\mathbf{d}_{+}$denotes the vector $\mathbf{d}_{k_{i}}$ corresponding to

$$
\max \left\{h\left(s_{9}\right), h\left(s_{10}\right), h\left(s_{11}\right), h\left(s_{12}\right), h\left(s_{13}\right), h\left(s_{14}\right), h\left(s_{15}\right), h\left(s_{16}\right)\right\}
$$

and $\mathbf{d}_{-}$denotes the vector $\mathbf{d}_{k_{i}}$ corresponding to

$$
\max \left\{h\left(s_{1}\right), h\left(s_{2}\right), h\left(s_{3}\right), h\left(s_{4}\right), h\left(s_{5}\right), h\left(s_{6}\right), h\left(s_{7}\right), h\left(s_{8}\right)\right\} .
$$

The LLR values for other bits $b_{k_{i}}^{1}, b_{k_{i}}^{2}, b_{k_{i}}^{3}$ can be derived similarly. Then we use Equations (7), (13), and (14) to convert LLRs to soft symbol estimate $\bar{d}_{k_{i}}$.

Table I shows the complexity comparison among different VBLAST detectors including:

1) VBLAST I - the conventional VBLAST expressed by (3).

2) VBLAST II - the improved detector using soft symbol estimate and nulling filter (introduced in Sec. III).

$z_{i}$ is derived using (5); $\mathbf{m}_{i}$ is derived using (6); LLRs are derived using (10) and (12).

3) VBLAST III - the improved detector with refined error criterion and nulling filter (introduced in Sec. IV).

$z_{i}$ is derived using (16); $\boldsymbol{w}_{i}$ is derived using (17); LLRs are derived using (28).

The comparison is made for an $N \times N$ MIMO configuration. The table shows the required number of complex operations including divisions, multiplications, and additions/subtractions for each symbol estimate. It can be seen from the table that the proposed schemes (especially the VBLAST-III) have a higher complexity than the 


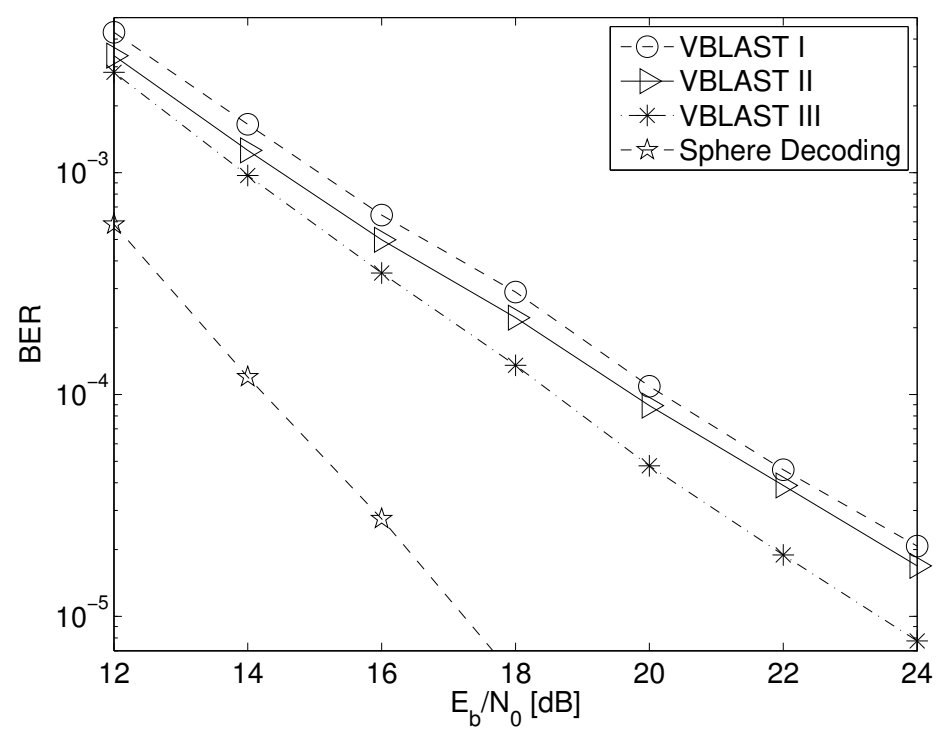

Fig. 3. Comparison of different VBLAST schemes in $4 \times 4$ QPSK systems.

conventional scheme. However, the complexity of all the detectors is of the same order, which is cubic in the number of antennas. As will become evident in the next section, the complexity increase by the proposed VBLAST detectors is largely compensated by the significant performance improvements.

\section{NumericAl Results}

Different VBLAST schemes are evaluated and compared through computer simulations. We assume an uncorrelated Rayleigh fading channel between each pair of transmit and receive antennas, and the channel is known to the receiver. The channel coefficients are normalized such that the average channel gain for each transmitted symbol is equal to unity. Fig. 3 shows the performance comparison of different VBLAST detectors in $4 \times 4$ QPSK systems. It can be seen from the figure that the improved detector VBLAST II outperforms the conventional detector VBLAST I. The conventional V-BLAST detector suffers from the error propagation problem, inaccurate replica miss-cancels the interference and errors would be propagated into the following interference cancellation stages. The VBLAST II detector alleviates this problem by using soft estimates of the CAI components for cancellation, while the VBLAST III detector further improves the performance by utilizing the complete second-order statistics, the performance advantage can be up to $2 \mathrm{~dB}$ compared to the conventional VBLAST detector. The gain is smaller at low SNRs due to the dominance of the circular channel noise. As SNR increases, the performance gain by the proposed detector becomes larger since it benefits more from the exploiting the noncircularity of the interference.

Fig. 3 also shows the near maximum likelihood performance obtained by the sphere decoding (SD), which performs a depth-first metric-constrained tree search on a triangular decomposition of the channel matrix [17]. The SD simulated here makes use of the Schnorr-Euchner enumeration [17], resulting in a lower complexity of the 


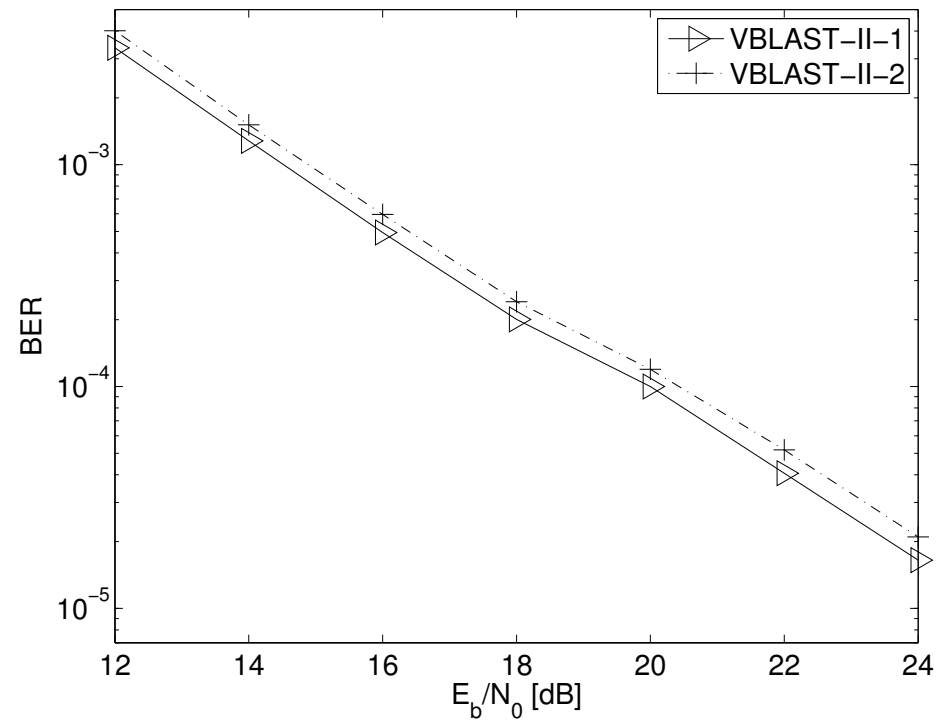

Fig. 4. VBLAST-II with and without nulling filter.

original SD. The initial radius in the SD is set to $\infty$ and reduced every time the tree search obtains a full-length path satisfying the metric constraint. It is evident from the figure that the peformance of the VBLAST I scheme is far away from the performance bound indicated by the SD curve, while the VBLAST III scheme brings the system closer to the bound by $2 \mathrm{~dB}$ as a result of alleviating the error propagation problem. It should be noted that the near-optimum performance produced by the SD comes at the cost of a high computational complexity which grows exponentially with block sizes and constellation orders [18].

In Fig. 4, we compare the performance of two implementations of VBLAST II algorithm: one with nulling filter (VBLAST-II-1); the other without nulling filtering (VBLAST-II-2). One can see from the figure that the detector with nulling filter performs slightly better than the one without, meaning that the nulling filter further suppresses the residual interference. Therefore, we only consider VBLAST-II-1 in the performance comparison conducted in this section.

Different VBLAST schemes with 16-QAM are examined in Figs. 5 and 6 for $4 \times 4$ and $8 \times 8$ systems, respectively. In the former case, the performance of the VBLAST II detector is almost identical to the conventional VBLAST detector, meaning that 16-QAM systems are more prone to error propagation problem, which cannot be effectively tackled just by replacing hard decision with soft symbol estimate. Fig. 5 also shows that the VBLAST-III detector performs significantly better than the others, which indicates that we need to use both soft symbol estimate and the complete second-order statistics in order to combat the error propagation inherent in the VBLAST detection process. Comparing Fig. 6 to Fig. 5, one can see that the performance gain achieved by the VBLAST III detector is more noticeable in $8 \times 8$ systems than in $4 \times 4$ systems. For example, a performance gain of up to $3 \mathrm{~dB}$ can 


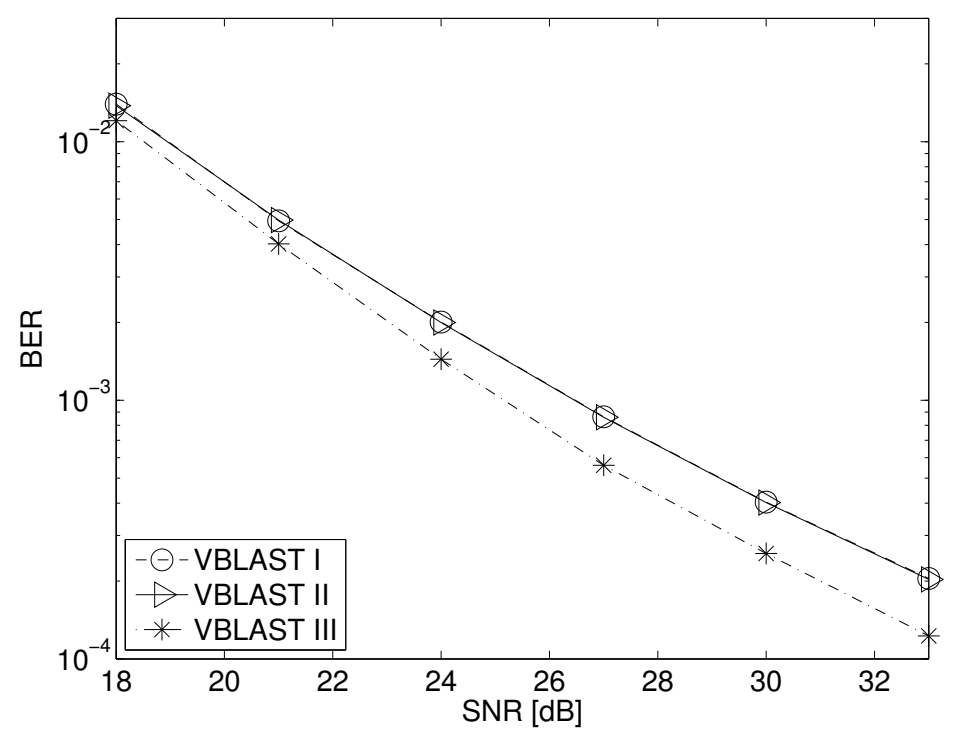

Fig. 5. Comparison of different VBLAST schemes in $4 \times 4$ 16-QAM systems.

be obtained by the VBLAST III detector compared to the conventional VBLAST detector.

\section{CONCLUSIONS}

Two variants of VBLAST detection algorithms for MIMO systems have been proposed. We showed that the conventional solution is suboptimal and its performance can be improved by utilizing soft symbol estimate and by refining the error criterion and nulling filter in such a way that the noncircular property of residula CAI and noise can be exploited. The proposed schemes are compared to the conventional V-BLAST scheme and are shown to achieve superior performance with moderate increase in the computational complexity.

\section{REFERENCES}

[1] E. Telatar. "Capacity of multi-antenna Gaussian channels". European Transactions on Telecommunications, vol. 10, no. 6, pp. 585-595, Nov. 1999.

[2] G. Foschini. "Layered space-time architecture for wireless communication in a fading environment when using multi-element antennas". Bell Labs Technical Journal, pp. 41-59, 1996.

[3] G. Golden, G. Foschini, R. Valenzuela, P. Wolniansky. "Detection algorithm and initial laboratory results using the V-BLAST space-time communication architecture". IEE Electronic Letters, vol. 35, no. 1, pp. 14-16, January 1999.

[4] P. Wolniansky, G. Foschini, G. Golden, R. Valenzuela. "V-BLAST: an architecture for realizing very high data rates over the richscattering wireless channel”. Proc. URSI International Symposium on Signals, Systems and Electronics, pp. 285-300, 1998.

[5] T. Ito, X. Wang, Y. Kakura, M. Madihian, A. Ushirokawa. "Performance comparison of MF and MMSE combined iterative soft interference canceller and V-BLAST technique in MIMO/OFDM systems”. Proc. IEEE Vehicular Technology Conference, vol. 1, pp. 488-492, 2003.

[6] T. Liu, Y. Liu. "Modified fast recursive algorithm for efficient MMSE-SIC detection of the V-BLAST system", IEEE Transactions on Wireless Communications, vol. 7, no. 10, pp. 3713-3717, Oct. 2008. 


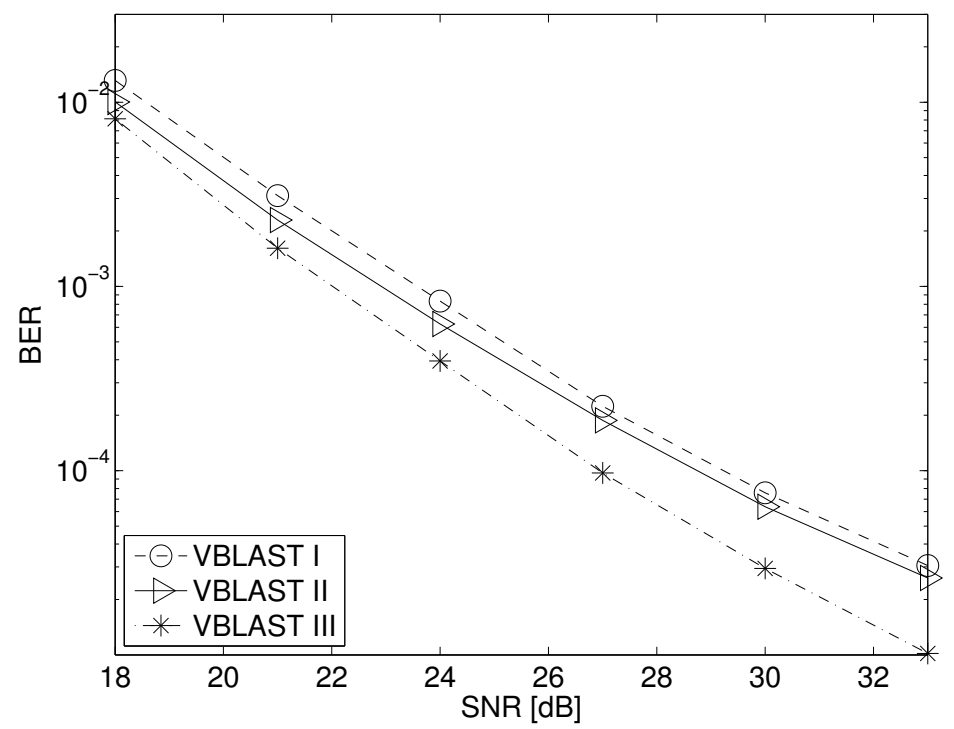

Fig. 6. Comparison of different VBLAST schemes in $8 \times 8$ 16-QAM systems.

[7] T. Liu. "Some results for the fast MMSE-SIC detection in spatially multiplexed MIMO systems", IEEE Transactions on Wireless Communications, vol. 8, no. 11, pp. 5443-5448, Nov. 2009.

[8] A. Alimohmmad, S. Fard, B. Cockburn. "Improved layered MIMO detection algorithm with near-optimal performance", IET Electronic Letters, vol. 45, no. 13, pp. 675-676, June 2009.

[9] J. Choi, H. Nguyen. "SIC-based detection with list and lattice redution for MIMO channels", IEEE Transactions on Vehicular Technology, vol. 58, no. 7, pp. 3786-3790, Sept. 2009.

[10] J. Choi, A. Singer, J. Lee, N. Cho. "Improved linear soft-input soft-output detection via soft feedback successive interference cancellation”, IEEE Transactions on Communications, vol. 58, no. 3, pp. 986-996, March 2010.

[11] S. L. Goff, A. Glavieux, C. Berrou. "Turbo-codes and high spectral efficiency modulation", IEEE International Conference on Communications, pp. 645-649, 1994.

[12] V. Poor, S. Verdu. "Probability of error in MMSE multiuser detection". IEEE Transactions on Communications, vol. 43, pp. 858-971, May 1997.

[13] X. Wautelet, A. Dejonghe, L. Vandendorpe. "MMSE-based fractional turbo receiver for space-time BICM over frequency-selective MIMO fading channels". IEEE Transactions on Signal Processing, vo. 52, no. 6, pp. 1804-1809, June 2004.

[14] P. Xiao, R. Liu. "Multi-user detector for multi-carrier CDMA systems". IET Electronic Letters, vol. 44, no. 23, pp. 1366-1368, Nov. 2008.

[15] G. Gelli, L. Paura, A. Ragozini. "Blind widely linear multiuser detection”. IEEE Communications Letters, vol. 4, no. 6, pp. 187-189, June 2000.

[16] S. Kay. Fundamentals of statistical signal processing, Prentice Hall, NJ, 1998.

[17] A. Murugan, H. Gamal, M. Damen, and G. Caire. "A unified framework for tree search decoding: rediscovering the sequential decoder”. IEEE Trans. on Inform. Theory, vol. 52, no. 3, pp. 933- 953, Mar. 2006.

[18] J. Jaldén and B. Ottersten. "On the complexity of sphere decoding in digital communications". IEEE Trans. on Sig. Proc., vol. 53, no. 4, pp. 1474-1484, Apr. 2005. 